PROCEEDINGS OF THE

AMERICAN MATHEMATICAL SOCIETY

Volume 134, Number 3, Pages 817-824

S 0002-9939(05)07992-X

Article electronically published on July 20, 2005

\title{
MULTIPLE POSITIVE SOLUTIONS OF SINGULAR PROBLEMS BY VARIATIONAL METHODS
}

\author{
RAVI P. AGARWAL, KANISHKA PERERA, AND DONAL O'REGAN \\ (Communicated by Carmen C. Chicone)
}

\begin{abstract}
The purpose of this paper is to use an appropriate variational framework to obtain positive solutions of some singular boundary value problems.
\end{abstract}

\section{INTRODUCTION}

Consider the boundary value problem

$$
\left\{\begin{array}{l}
-y^{\prime \prime}=f(t, y), \quad 0<t<1, \\
y(0)=y(1)=0
\end{array}\right.
$$

where we only assume that $f \in C((0,1) \times(0, \infty),[0, \infty))$ satisfies

$$
2 \varepsilon \leq f(t, y) \leq C y^{-\gamma}, \quad(t, y) \in(0,1) \times(0, \varepsilon),
$$

for some $\varepsilon, C>0$ and $\gamma \in(0,1)$, so that it may be singular at $y=0$ (here of course $C$ could depend on $\varepsilon)$. A typical example is

$$
f(t, y)=y^{-\gamma}+g(t, y)
$$

with $g \in C((0,1) \times[0, \infty),[0, \infty))$.

Define $f_{\varepsilon} \in C((0,1) \times \mathbb{R},[0, \infty))$ by

$$
f_{\varepsilon}(t, y)=f\left(t,\left(y-\varphi_{\varepsilon}(t)\right)^{+}+\varphi_{\varepsilon}(t)\right)
$$

where $\varphi_{\varepsilon}(t)=\varepsilon t(1-t)$ is the solution of

$$
\left\{\begin{array}{l}
-y^{\prime \prime}=2 \varepsilon, \quad 0<t<1, \\
y(0)=y(1)=0
\end{array}\right.
$$

and $y^{ \pm}=\max \{ \pm y, 0\}$, and consider

$$
\left\{\begin{array}{l}
-y^{\prime \prime}=f_{\varepsilon}(t, y), \quad 0<t<1, \\
y(0)=y(1)=0 .
\end{array}\right.
$$

By (1.2),

$$
2 \varepsilon \leq f_{\varepsilon}(t, y) \leq C \varphi_{\varepsilon}(t)^{-\gamma}, \quad(t, y) \in(0,1) \times(-\infty, \varepsilon) .
$$

Received by the editors July 22, 2004 and, in revised form, October 20, 2004. 2000 Mathematics Subject Classification. Primary 34B15, 34B16.

Key words and phrases. Singular, boundary value problem, variational, positive, multiple. 
We observe that if $y$ is a solution of (1.6), then $y \geq \varphi_{\varepsilon}$ and hence also a solution of (1.1). To see this suppose

$$
y(t)<\varphi_{\varepsilon}(t) \text { for some } t .
$$

By Lemma 2.8.1 of Agarwal and O'Regan [1],

$$
y(t) \geq t(1-t)|y|_{0}, \quad t \in[0,1],
$$

where $|y|_{0}=\max _{t \in[0,1]}|y(t)|$, so (1.8) implies $|y|_{0}<\varepsilon$. But then $-y^{\prime \prime} \geq 2 \varepsilon=-\varphi_{\varepsilon}^{\prime \prime}$ by (1.7), so $y \geq \varphi_{\varepsilon}$, contradicting (1.8). Conversely, every solution of (1.1) is a solution of (1.6).

Since $\varphi_{\varepsilon}^{-\gamma} \in L^{1}(0,1)$, we see from (1.7) that solutions of (1.6) are the critical points of the $C^{1}$ functional

$$
\Phi(y)=\int_{0}^{1}\left(\frac{1}{2}\left|y^{\prime}(t)\right|^{2}-F_{\varepsilon}(t, y(t))\right) d t, \quad y \in H=H_{0}^{1}(0,1),
$$

where $F_{\varepsilon}(t, y)=\int_{\varepsilon}^{y} f_{\varepsilon}(t, x) d x$ and $H_{0}^{1}(0,1)$ is the usual Sobolev space, normed by

$$
\|y\|=\left(\int_{0}^{1}\left|y^{\prime}(t)\right|^{2} d t\right)^{1 / 2}
$$

The purpose of this paper is to use this variational framework to obtain positive solutions of (1.1).

We will show that, under additional assumptions on the behavior of $f$ at infinity, $\Phi$ satisfies the compactness condition of Cerami [2]:

(C): every sequence $\left\{y_{m}\right\} \subset H$ such that

$$
\Phi\left(y_{m}\right) \text { is bounded, } \quad\left(1+\left\|y_{m}\right\|\right)\left\|\Phi^{\prime}\left(y_{m}\right)\right\| \rightarrow 0
$$

has a convergent subsequence.

This condition is weaker than the usual Palais-Smale condition, but can be used in place of it when constructing deformations of sublevel sets via negative pseudogradient flows, and therefore also in minimax theorems such as the mountain pass lemma.

By a standard argument it suffices to show that $\left\{y_{m}\right\}$ is bounded when verifying (C). Moreover,

$$
\begin{aligned}
\left\|y_{m}^{-}\right\|^{2} & =-\left(\int_{0}^{1} f_{\varepsilon}\left(t, y_{m}(t)\right) y_{m}^{-}(t) d t+\left\langle\Phi^{\prime}\left(y_{m}\right), y_{m}^{-}\right\rangle\right) \\
& \leq\left|y_{m}^{-}\right|_{0} \int_{y_{m}<0} f_{\varepsilon}\left(t, y_{m}(t)\right) d t+\left\|\Phi^{\prime}\left(y_{m}\right)\right\|\left\|y_{m}^{-}\right\|
\end{aligned}
$$

and hence

$$
\left\|y_{m}^{-}\right\| \leq C \int_{0}^{1} \varphi_{\varepsilon}(t)^{-\gamma} d t+o(1)
$$

since $|\cdot|_{0} \leq\|\cdot\|$ and by (1.7), so we will only need to check that $\left\{y_{m}^{+}\right\}$is bounded.

We refer the reader to Agarwal and O'Regan 11 for a broad introduction to singular problems and to Rabinowitz [3] for variational methods. 


\section{An EXISTENCE PRINCIPLE}

Assume

(f): there is a constant $M>0$, independent of $\lambda$, such that $\|y\| \neq M$ for every solution $y>0$ to

$$
\left\{\begin{array}{l}
-y^{\prime \prime}=\lambda f(t, y), \quad 0<t<1 \\
y(0)=y(1)=0
\end{array}\right.
$$

for each $\lambda \in(0,1]$.

Note that (f) holds if there exists an a priori bound of the norm of the solutions of the problem.

Proposition 2.1. If (1.2) and (f) hold, then (1.1) has a positive solution.

Proof. We will show that $\Phi$ assumes its infimum on

$$
B=\{y \in H:\|y\| \leq M\}
$$

at some point $y_{0} \in \stackrel{\circ}{B}$, which is then a local minimizer, if $\varepsilon$ is chosen small enough.

Clearly, $\inf \Phi(B)>-\infty$. Let $\left\{y_{m}\right\}$ be a minimizing sequence. Passing to a subsequence we may assume that $y_{m}$ converges to some $y_{0} \in B$ weakly in $H$, strongly in $L^{2}(0,1)$, and a.e. in $(0,1)$. Then

$$
\begin{aligned}
\Phi\left(y_{0}\right) & =\frac{1}{2}\left\|y_{0}\right\|^{2}-\int_{0}^{1} F_{\varepsilon}\left(t, y_{0}(t)\right) d t \\
& \leq \liminf \frac{1}{2}\left\|y_{m}\right\|^{2}-\lim \int_{0}^{1} F_{\varepsilon}\left(t, y_{m}(t)\right) d t \\
& =\lim \Phi\left(y_{m}\right)=\inf _{y \in B} \Phi(y),
\end{aligned}
$$

so $\Phi\left(y_{0}\right)=\inf \Phi(B)$.

Suppose that $y_{0} \in \partial B$. Then it is also a minimizer of $\left.\Phi\right|_{\partial B}$, so the gradient of $\Phi$ at $y_{0}$ points in the direction of the inward normal to $\partial B$, i.e.,

$$
\Phi^{\prime}\left(y_{0}\right)=-\nu y_{0}
$$

or

$$
-y_{0}^{\prime \prime}=\frac{1}{1+\nu} f_{\varepsilon}\left(t, y_{0}\right)
$$

for some $\nu \geq 0$. If $y_{0} \geq \varphi_{\varepsilon}$, (2.5) reduces to (2.1) with $\lambda=\frac{1}{1+\nu} \in(0,1]$, so, as in the introduction, it follows that $y_{0}<\varepsilon$. But then multiplying (2.5) by $y_{0}$ and integrating by parts gives

$$
M^{2}=\frac{1}{1+\nu} \int_{0}^{1} y_{0}(t) f_{\varepsilon}\left(t, y_{0}(t)\right) d t \leq C \varepsilon \int_{0}^{1} \varphi_{\varepsilon}(t)^{-\gamma} d t=C \varepsilon^{1-\gamma}
$$

by (1.7), where $C$ is a generic positive constant, which is impossible if $\varepsilon$ is sufficiently small.

For example, consider

$$
\left\{\begin{array}{l}
-y^{\prime \prime}=\mu f(t, y), \quad 0<t<1 \\
y(0)=y(1)=0
\end{array}\right.
$$


where $\mu>0$ is a parameter and $f \in C((0,1) \times(0, \infty),[0, \infty))$ satisfies (1.2). If $y>0$ is a solution of

$$
\left\{\begin{array}{l}
-y^{\prime \prime}=\lambda \mu f(t, y), \quad 0<t<1, \\
y(0)=y(1)=0
\end{array}\right.
$$

with $\|y\|=M$,

$$
M^{2}=\lambda \mu \int_{0}^{1} y(t) f(t, y(t)) d t \leq \mu \sup _{(t, y) \in(0,1) \times(0, M]} y f(t, y),
$$

so (2.7) has a positive solution for

$$
\mu<\sup _{M>0} \frac{M^{2}}{\sup _{(t, y) \in(0,1) \times(0, M]} y f(t, y)} .
$$

Similarly,

$$
\left\{\begin{array}{l}
-y^{\prime \prime}=y^{-\gamma}+\mu g(t, y), \quad 0<t<1, \\
y(0)=y(1)=0
\end{array}\right.
$$

where $\gamma \in(0,1), \mu>0$ is a parameter, and $g \in C((0,1) \times[0, \infty),[0, \infty))$ has a positive solution for

$$
\mu<\sup _{M>0} \frac{M^{1-\gamma}\left(M^{1+\gamma}-1\right)}{\sup _{(t, y) \in(0,1) \times(0, M]} y g(t, y)} .
$$

\section{Asymptotically Linear CASE}

Assume

$$
f(t, y) \leq C y, \quad(t, y) \in(0,1) \times[\varepsilon, \infty),
$$

for some $C>0$. We say that (1.1) is resonant if

$$
\frac{f(t, y)}{y} \rightarrow \lambda_{1} \quad \text { as } y \rightarrow \infty
$$

where $\lambda_{1}=\pi^{2}$ is the first eigenvalue of

$$
\left\{\begin{array}{l}
-y^{\prime \prime}=\lambda y, \quad 0<t<1 \\
y(0)=y(1)=0
\end{array}\right.
$$

Denote by

$$
H(t, y)=F_{\varepsilon}(t, y)-\frac{1}{2} y f_{\varepsilon}(t, y)
$$

the nonquadratic part of $F_{\varepsilon}$.

Theorem 3.1. If (1.2) and (3.1) hold, then (1.1) has a positive solution in the following cases:

(i) Nonresonance below $\lambda_{1}$ :

$$
f(t, y) \leq a y+C, \quad(t, y) \in(0,1) \times[\varepsilon, \infty),
$$

for some $a<\lambda_{1}$ and $C>0$, 
(ii) Resonance: (3.2) holds,

$$
H(t, y) \leq C, \quad(t, y) \in(0,1) \times[\varepsilon, \infty),
$$

for some $C>0$, and

$$
H(t, y) \rightarrow-\infty \quad \text { as } y \rightarrow \infty .
$$

Proof. (i) By (1.7) and (3.5),

$$
F_{\varepsilon}(t, y) \leq \begin{cases}0, & y<\varepsilon, \\ \frac{a}{2} y^{2}+C y, & y \geq \varepsilon,\end{cases}
$$

and, since $a<\lambda_{1}$, it follows from Wirtinger's inequality that $\Phi$ is bounded from below and coercive, and hence satisfies (C) and admits a global minimizer.

(ii) For $y \geq \varepsilon$,

$$
\frac{\partial}{\partial y}\left(\frac{F_{\varepsilon}(t, y)}{y^{2}}\right)=-\frac{2 H(t, y)}{y^{3}}, \quad \frac{F_{\varepsilon}(t, y)}{y^{2}} \rightarrow \frac{\lambda_{1}}{2} \quad \text { as } y \rightarrow \infty
$$

by (3.2), so

$$
F_{\varepsilon}(t, y)=\left(\frac{\lambda_{1}}{2}+2 \int_{y}^{\infty} \frac{H(t, x)}{x^{3}} d x\right) y^{2} \leq \frac{\lambda_{1}}{2} y^{2}+C
$$

by (3.6), and hence Wirtinger's inequality implies $\Phi$ is bounded from below.

To verify (C), let $\left\{y_{m}\right\}$ satisfy (1.12) and suppose $\rho_{m}:=\left\|y_{m}\right\| \rightarrow \infty$. Since $\left\{y_{m}^{-}\right\}$ is bounded, for a subsequence, $\widetilde{y}_{m}:=\frac{y_{m}}{\rho_{m}}$ converges to some $\widetilde{y} \geq 0$ weakly in $H$, strongly in $L^{2}(0,1)$, and a.e. in $(0,1)$. Then

$$
\int_{0}^{1} \widetilde{y}_{m}^{\prime}(t)\left(\widetilde{y}_{m}^{\prime}(t)-\widetilde{y}^{\prime}(t)\right) d t=\int_{0}^{1} g_{m}(t) d t+\frac{\left\langle\Phi^{\prime}\left(y_{m}\right), \widetilde{y}_{m}-\widetilde{y}\right\rangle}{\rho_{m}}
$$

where $g_{m}(t)=\frac{f\left(t, y_{m}(t)\right)}{\rho_{m}}\left(\widetilde{y}_{m}(t)-\widetilde{y}(t)\right)$. By (1.7) and (3.1),

$$
\left|g_{m}(t)\right| \leq C\left(\varphi_{\varepsilon}(t)^{-\gamma}+1\right)\left|\widetilde{y}_{m}(t)-\widetilde{y}(t)\right|
$$

and hence $g_{m} \rightarrow 0$ a.e. and $\left|g_{m}\right| \leq C\left(\varphi_{\varepsilon}^{-\gamma}+1\right) \in L^{1}(0,1)$, so passing to the limit in (3.11) gives $\|\widetilde{y}\|=1$; in particular, $\widetilde{y} \neq 0$.

By (1.7) and (3.6),

$$
H(t, y) \leq \begin{cases}C \varphi_{\varepsilon}(t)^{-\gamma}|y|, & y<0, \\ 0, & 0 \leq y<\varepsilon, \\ C, & y \geq \varepsilon,\end{cases}
$$

and $\left|y_{m}^{-}\right|_{0}$ is bounded, so

$$
\int_{\widetilde{y}>0} H\left(t, y_{m}(t)\right) d t \rightarrow-\infty
$$


by (3.7) and $\int_{\widetilde{y}=0} H\left(t, y_{m}(t)\right) d t$ is bounded from above. Hence

$$
\begin{aligned}
\frac{1}{2}\left\langle\Phi^{\prime}\left(y_{m}\right), y_{m}\right\rangle & -\Phi\left(y_{m}\right)=\int_{0}^{1} H\left(t, y_{m}(t)\right) d t \\
& =\int_{\widetilde{y}>0} H\left(t, y_{m}(t)\right) d t+\int_{\widetilde{y}=0} H\left(t, y_{m}(t)\right) d t \rightarrow-\infty,
\end{aligned}
$$

contrary to assumption.

Theorem 3.2. If (1.2), (3.1), and (f) hold, then (1.1) has two positive solutions in the following cases:

(i) Resonance: (3.2) holds,

$$
H(t, y) \geq-C, \quad(t, y) \in(0,1) \times[\varepsilon, \infty)
$$

for some $C>0$, and

$$
H(t, y) \rightarrow+\infty \quad \text { as } y \rightarrow \infty
$$

(ii) Nonresonance above $\lambda_{1}$ :

$$
f(t, y) \geq b y-C, \quad(t, y) \in(0,1) \times[\varepsilon, \infty),
$$

for some $b>\lambda_{1}$ and $C>0$.

Proof. By (see the proof of) Proposition 2.1, $\Phi$ has a local minimizer $y_{0} \in \stackrel{\circ}{B}$ and $\inf \Phi(\partial B) \geq \Phi\left(y_{0}\right)$. We will show that $\Phi\left(R \varphi_{1}\right) \leq \inf \Phi(\partial B)$ if $R>M$ is sufficiently large, where $\varphi_{1}>0$ is the normalized eigenfunction associated with $\lambda_{1}$, and we will verify $(\mathrm{C})$. Then the mountain pass lemma will give a second critical point at the level

$$
c:=\inf _{\gamma \in \Gamma} \max _{y \in \gamma([0,1])} \Phi(y)
$$

where

$$
\Gamma=\left\{\gamma \in C([0,1], H): \gamma(0)=y_{0}, \gamma(1)=R \varphi_{1}\right\}
$$

is the class of paths joining $y_{0}$ and $R \varphi_{1}$.

(i) By (3.10), (3.16), and (3.17),

$$
\begin{aligned}
\frac{\lambda_{1}}{2} y^{2}-F_{\varepsilon}(t, y)=-2 y^{2} \int_{y}^{\infty} \frac{H(t, x)}{x^{3}} d x \leq C, \quad y \geq \varepsilon & \text { and } \rightarrow-\infty \quad \text { as } y \rightarrow \infty,
\end{aligned}
$$

and by (1.7)

$$
\frac{\lambda_{1}}{2} y^{2}-F_{\varepsilon}(t, y) \leq C\left(\varphi_{\varepsilon}(t)^{-\gamma}+1\right), \quad 0 \leq y<\varepsilon,
$$

so

$$
\Phi\left(R \varphi_{1}\right)=\int_{0}^{1}\left(\frac{\lambda_{1}}{2} R^{2} \varphi_{1}(t)^{2}-F_{\varepsilon}\left(t, R \varphi_{1}(t)\right)\right) d t \rightarrow-\infty \quad \text { as } R \rightarrow \infty .
$$

The verification of $(\mathrm{C})$ is similar to that in the proof of Theorem 3.1 
(ii) By (1.7) and (3.18),

$$
F_{\varepsilon}(t, y) \geq \begin{cases}-C \varphi_{\varepsilon}(t)^{-\gamma}, & 0 \leq y<\varepsilon, \\ \frac{b}{2} y^{2}-C y, & y \geq \varepsilon,\end{cases}
$$

and, since $b>\lambda_{1}$, it follows that $\Phi\left(R \varphi_{1}\right) \rightarrow-\infty$ as $R \rightarrow \infty$.

If (1.12) holds and $\rho_{m}:=\left\|y_{m}\right\| \rightarrow \infty$, passing to a subsequence $\widetilde{y}_{m}:=\frac{y_{m}}{\rho_{m}}$ converges to a nontrivial $\widetilde{y} \geq 0$ weakly in $H$, strongly in $L^{2}(0,1)$, and a.e. in $(0,1)$ as in the proof of Theorem 3.1 Then

$$
\begin{aligned}
\left(b-\lambda_{1}\right) & \int_{0}^{1} \widetilde{y}_{m}(t) \varphi_{1}(t) d t=\frac{1}{\rho_{m}} \int_{0}^{1}\left(b y_{m}(t) \varphi_{1}(t)-y_{m}^{\prime}(t) \varphi_{1}^{\prime}(t)\right) d t \\
& =\frac{1}{\rho_{m}}\left(\int_{0}^{1}\left(b y_{m}(t)-f_{\varepsilon}\left(t, y_{m}(t)\right)\right) \varphi_{1}(t) d t-\left\langle\Phi^{\prime}\left(y_{m}\right), \varphi_{1}\right\rangle\right) \\
& \leq \frac{1}{\rho_{m}}\left(\int_{y_{m} \geq \varepsilon} C \varphi_{1}(t) d t+\int_{y_{m}<\varepsilon} b y_{m}(t) \varphi_{1}(t) d t+\left\|\Phi^{\prime}\left(y_{m}\right)\right\|\right)
\end{aligned}
$$

by (3.18), and $\left|y_{m}^{-}\right|_{0}$ is bounded, so

$$
\left(b-\lambda_{1}\right) \int_{0}^{1} \widetilde{y}(t) \varphi_{1}(t) d t \leq 0,
$$

which is impossible.

\section{SuperLinear CASE}

Assume

$$
0<\theta F_{\varepsilon}(t, y) \leq y f(t, y), \quad(t, y) \in(0,1) \times\left[y_{0}, \infty\right),
$$

for some $\theta>2$ and $y_{0}>\varepsilon$.

Theorem 4.1. If (1.2), (4.1), and (f) hold, then (1.1) has two positive solutions.

Proof. As in the proof of Theorem 3.2 it suffices to show that $\Phi\left(R \varphi_{1}\right) \rightarrow-\infty$ as $R \rightarrow \infty$ and to verify (C). The former follows since

$$
F_{\varepsilon}(t, y) \geq \begin{cases}-C \varphi_{\varepsilon}(t)^{-\gamma}, & 0 \leq y<\varepsilon, \\ F_{\varepsilon}\left(t, y_{0}\right)\left(\frac{y}{y_{0}}\right)^{\theta}, & y \geq y_{0},\end{cases}
$$

by (1.7) and (4.1). As for the latter,

$$
\begin{aligned}
\left(\frac{\theta}{2}-1\right)\left\|y_{m}\right\|^{2}= & \int_{0}^{1}\left(\theta F_{\varepsilon}\left(t, y_{m}(t)\right)-y_{m}(t) f_{\varepsilon}\left(t, y_{m}(t)\right)\right) d t \\
& +\theta \Phi\left(y_{m}\right)-\left\langle\Phi^{\prime}\left(y_{m}\right), y_{m}\right\rangle \\
\leq & C\left(\int_{y_{m}<\varepsilon} \varphi_{\varepsilon}(t)^{-\gamma}\left|y_{m}(t)\right| d t+1\right)
\end{aligned}
$$

and the last integral is bounded since $\left|y_{m}^{-}\right|_{0}$ is bounded. 
For example, Theorem 4.1 guarantees that

$$
\left\{\begin{array}{l}
-y^{\prime \prime}=\mu\left(y^{-\gamma}+y^{\beta}\right), \quad 0<t<1, \\
y(0)=y(1)=0
\end{array}\right.
$$

where $\gamma \in(0,1)$ and $\beta>1$, has two positive solutions for

$$
0<\mu<\frac{\left((\gamma+1)^{\gamma+1}(\beta-1)^{\beta-1}\right)^{1 /(\gamma+\beta)}}{\gamma+\beta}
$$

(see the example after Proposition 2.1 and $(2.10)$ ). The problem

$$
\left\{\begin{array}{l}
-y^{\prime \prime}=y^{-\gamma}+\mu e^{y}, \quad 0<t<1, \\
y(0)=y(1)=0
\end{array}\right.
$$

where $\gamma \in(0,1)$, has two positive solutions for

$$
0<\mu<\sup _{M>0} \frac{M^{1+\gamma}-1}{M^{\gamma} e^{M}}
$$

$($ see $(2.12))$.

\section{REFERENCES}

[1] Ravi P. Agarwal and Donal O'Regan. Singular differential and integral equations with applications. Kluwer Academic Publishers, Dordrecht, 2003. MR2011127 (2004h:34002)

[2] Giovanna Cerami. An existence criterion for the critical points on unbounded manifolds. Istit. Lombardo Accad. Sci. Lett. Rend. A, 112(2):332-336 (1979), 1978. MR0581298 (81k:58021)

[3] Paul H. Rabinowitz. Minimax methods in critical point theory with applications to differential equations, volume 65 of CBMS Regional Conference Series in Mathematics. Published for the Conference Board of the Mathematical Sciences, Washington, DC, 1986. MR0845785 $(87 \mathrm{j}: 58024)$

Department of Mathematical Sciences, Florida Institute of Technology, Melbourne, FLORIDA 32901

Department of Mathematical Sciences, Florida Institute of Technology, Melbourne, FLORIDA 32901

Department of Mathematics, National University of Ireland, Galway, Ireland 